Meta

Journal des traducteurs

Translators' Journal

\title{
Présentation
}

\section{Claude Tatilon}

Volume 25, numéro 4, décembre 1980

URI : https://id.erudit.org/iderudit/004325ar

DOI : https://doi.org/10.7202/004325ar

Aller au sommaire du numéro

Éditeur(s)

Les Presses de l'Université de Montréal

ISSN

0026-0452 (imprimé)

1492-1421 (numérique)

Découvrir la revue

Citer ce document

Tatilon, C. (1980). Présentation. Meta, 25(4), 391-392.

https://doi.org/10.7202/004325ar

Ce document est protégé par la loi sur le droit d'auteur. L'utilisation des services d'Érudit (y compris la reproduction) est assujettie à sa politique d'utilisation que vous pouvez consulter en ligne.

https://apropos.erudit.org/fr/usagers/politique-dutilisation/
Cet article est diffusé et préservé par Érudit.

Érudit est un consortium interuniversitaire sans but lucratif composé de l’Université de Montréal, l'Université Laval et l'Université du Québec à Montréal. Il a pour mission la promotion et la valorisation de la recherche. https://www.erudit.org/fr/ 


\section{Présentation}

Claude Tatilon

Admettons-le tout de suite: en matière de traduction, la pratique est première - primum traducere deinde philosophari, en quelque sorte. Pourtant, les mots qui suivent, écrits en préambule aux textes des communications, vont immédiatement prendre l'allure d'un plaidoyer pro theoria. On pourrait nous le reprocher. Comme on pourrait nous reprocher le parti pris de notre dépliant annonçant le colloque, où nous fustigions un traducteur imaginaire, enlisé dans son travail quotidien, lui faisant prononcer ces paroles à l'emportepièce :

Ça fait plus de 30 ans, jeune homme, que j'exerce ce métier. Je suis un praticien «pure laine», moi... et fier de l'être : jamais une seule fois je n'ai eu à rougir de mon travail. Et pourtant, laissez-moi vous dire, j'ignore tout de la linguistique, de la stylistique et autres foutaises universitaires. Alors, la THÉ-O-RIE, moi, vous comprenez...

Le paradoxe n'est qu'apparent : pour la théorie, oui! Mais dans la mesure où elle sert, où elle tonifie la pratique...

Cette assistance de la théorie à la pratique, nous allons l'illustrer par deux exemples. Le premier, emprunté à la publicité canadienne, est celui-ci :

\section{Tea. Iced'n easy. \\ T'as chaud? Thé glacé!}

Dans cette traduction réussie, on jugera parfaite l'équivalence des deux slogans, du moins si on l'entend au sens pragmatique : en effet, les slogans sont d'une efficacité publicitaire comparable ou, dirons-nous en termes plus techniques, présentent les mêmes fonctions : une fonction identificatrice (Iced tea - thé glacé), une fonction laudative, implicite («agréable», «rafraîchissant»), une fonction ludique (les deux jeux de mots) et une fonction mnémotechnique (concision et humour). Devant cette réussite, deux conclusions théoriques s'imposent :

$1^{\circ}$ - Traduire est un acte de communication dont le succès se mesure à son efficacité pratique. (On remarquera à ce propos l'insistance de plusieurs conférenciers sur la notion de destinataire.)

$2^{\circ}-$ Traduire consiste à reproduire dans le texte d'arrivée l'information pertinente du texte de départ, - et non les structures porteuses de cette information.

Le second exemple est celui d'un échec. Il va nous permettre d'insister un peu plus sur la notion, théoriquement fondamentale, de pertinence. À l'article Synonymes de la version française d'une encyclopédie américaine destinée à 
de jeunes lecteurs, on trouve, à la suite d'une définition acceptable de la synonymie, l'exemple suivant :

$$
\text { ex. : petit et faible }
$$

Perplexité du lecteur, auquel n'échappe pas... la faiblesse de l'illustration. La clé du mystère est à chercher dans le texte américain, qui propose en exemple :

$$
\text { e.g. : small - little }
$$

Dès lors, tout s'éclaire. Manquant de lumières théoriques, le traducteur a succombé à ce que Jean Darbelnet appelle avec justesse «la tyrannie de la forme». L'information pertinente ici ne résidait pas dans les signifiés du couple de lexèmes (qui ne se retrouve pas dans le lexique français) mais dans le rapport synonymique les unissant, - rapport que n'importe quelle paire de synonymes aurait suffi à illustrer: vélo - bicyclette, auto - voiture, boisson - breuvage, patate - pomme de terre...

«La théorie au service de la pratique», donc. Mais dans cet ordre, non dans l'ordre inverse. Parce qu'en matière de traduction, le plus important, répétons-le, c'est bien la pratique.

Qu'il me soit permis, en terminant, d'adresser des remerciements personnels aux trois autres membres de notre comité d'organisation: Yvette Szmidt, Alain Baudot, Howard Robertson, de chacun desquels je puis dire sans la moindre hésitation qu'il/elle a fait un bon quart du travail.

Qu'il me soit permis encore, au nom de nous quatre, de remercier toutes les bonnes volontés glendoniennes - trop nombreuses pour être nommées qui se sont très efficacement mises «au service du colloque».

Je n'aurai pas, enfin, la suprême ingratitude de passer sous silence les noms suivants :

l'Association des traducteurs et interprètes de l'Ontario;

le Conseil de recherches en sciences humaines du Canada;

le ministère des Affaires extérieures, Canada;

le ministère des Affaires culturelles et des Loisirs, Ontario;

le ministère des Collèges et Universités, Division des Affaires universitaires, Ontario ;

le ministère de l'Éducation nationale et de la Culture néerlandaise, Belgique ;

Pro Helvetia, Suisse;

le Secrétariat d'État, Bureau des traductions, Canada;

les Services culturels français, Toronto;

l'Université York, Toronto;

Wintario.

Sans l'aide généreuse de ces organismes, nous n'aurions simplement pas pu mener à bien notre entreprise. Qu'il me soit donc aussi permis de leur présenter, au nom de tous les participants, nos remerciements les plus vifs. 